\title{
Alter
}

Revue de phénoménologie

$21 \mid 2013$

La Vie

\section{La question du corps vivant (Leib) chez Heidegger, des Zollikoner Seminare À Sein und Zeit et retour}

\section{Christian Sommer}

\section{(2) OpenEdition}

Journals

Édition électronique

URL : http://journals.openedition.org/alter/851

DOI : $10.4000 /$ alter.851

ISSN : 2558-7927

Éditeur :

Association ALTER, Archives Husserl (CNRS-UMR 8547)

Édition imprimée

Date de publication : 1 novembre 2013

Pagination : 205-220

ISBN : 978-2-95-223749-9

ISSN : 1249-8947

Référence électronique

Christian Sommer, « La question du corps vivant (Leib) chez Heidegger, des Zollikoner Seminare À Sein und Zeit et retour », Alter [En ligne], 21 | 2013, mis en ligne le 01 juin 2019, consulté le 06 juillet 2019.

URL : http://journals.openedition.org/alter/851 ; DOI : 10.4000/alter.851

Revue Alter 


\section{LA QUESTION DU CORPS VIVANT (LEIB) CHEZ HEIDEGGER, DES ZOLLIKONER SEMINARE À SEIN UND ZEIT ET RETOUR ${ }^{1}$}

Christian Sommer

Dans l'entretien avec M. Boss du 8 juillet 1965, Heidegger glose l'une des définitions aristotéliciennes de l'âme: "Chez Aristote, la psyché (Psyche) est l'entéléchie du corps vivant (Leib) ou du sôma. Dans son livre De anima, il dit (412a) : la psyché est le mode de l'être d'un vivant (Die Psyche ist die Weise des Seins eines Lebendigen) ${ }^{2}$. Et Heidegger ajoute : «L'entéléchie de l'homme est le logos »33. Or dans le christianisme, dans la scolastique latine, poursuit Heidegger dans son entretien, « la psyché est réifiée (verdinglicht) ; elle n'est plus un mode d'être du vivant, mais devient quelque chose, devient une substance psychique (Seelensubstanz) $»^{4}$. C'est donc dans le christianisme que s'opère selon Heidegger la funeste substitution de corpus à sôma, d'ailleurs à l'origine du supposé aveuglement de la Leibesphänomenologie française des années 1950 et 1960, phénoménologie française qui serait dès lors réduite à penser le phénomène du Leib dans le cadre restreint du corpus, et impuissante par là même à saisir la « véritable

\footnotetext{
${ }^{1}$ Cet article reprend une communication donnée le 18 juin 2011 à l'invitation de Philippe Cabestan dans le cadre d'une journée d'étude sur «Martin Heidegger et les Séminaires de Zollikon » organisée par la Société Française de Daseinsanalyse à l'Université de Paris-I.

${ }^{2}$ Cf. Aristote, De an., II, 1, 412a27 : «L'âme est l'entéléchie première d'un corps naturel qui est potentiellement vivant». Les traductions d'Aristote, parfois modifiées, sont généralement empruntées aux traductions de J. Tricot (Vrin). Pour le Traité de l'âme, on consultera aussi la traduction récente par R. Bodéüs (GF).

${ }^{3}$ Heidegger, Zollikoner Seminare. Protokolle - Gespräche - Briefe (1959-1969), Francfort-sur-leMain, Klostermann, 1987, p. 250. Pour homogénéiser la terminologie, nous traduisons toutes les citations de Heidegger (généralement d'après la Gesamtausgabe [GA], Francfort-sur-le-Main, Klostermann, 1975 et suiv.), y compris celles tirées des Zollikoner Seminare, curieusement traduits en français sous le titre Séminaires de Zurich (trad. C. Gros, Paris, Gallimard, 2010).

${ }^{4}$ Heidegger, Zollikoner Seminare, p. 250. Cf. déjà Grundbegriffe der antiken Philosophie (1926), GA 22, p. 184.
} 
problématique d'une phénoménologie du Leib $»^{5}$. Lors de la séance du 14 mai 1965, Heidegger, en effet, n’hésite pas à expliquer à ses auditeurs :

\begin{abstract}
Contre la distinction entre corps (Körper) et corps vivant (Leib), on entend souvent l'objection qu'il y a là un problème, quelque part, puisque, par exemple, les Français n'ont aucun mot pour Leib, mais seulement un nom pour Körper: "le corps". Or, qu'est-ce que cela signifie? Cela signifie que dans ce domaine, les Français sont exclusivement déterminés par le corpus latin; cela signifie encore que la tâche de voir la véritable problématique de la Leibesphänomenologie leur est rendue fort difficile. La significaction du mot grec «sôma » est très diverse [...] "sôma" possède en grec une signification bien plus large que notre actuel "somatique ». Très généralement on peut dire qu'on a réinterprété le grec à partir du latin. Notre représentation (Vorstellung) du corps nous vient du latin corpus. Selon la représentation scolastique, le Leib est un corps animé (beseelter Körper), une détermination qui, en un certain sens, remonte à Aristote, mais seulement en un certain sens ${ }^{6}$.
\end{abstract}

Nous voudrions interroger les implications de cette définition de l'âme dans la lecture heideggérienne d'Aristote telle qu'elle s'est effectuée dans les cours ou séminaires sur Aristote (1921 à 1926), mais aussi dans Sein und Zeit même, où les retombées de cette lecture ou "destruction » phénoménologique sont perceptibles, bien que transformées dans le vocabulaire de l'analytique du Dasein. C'est donc une certaine hypothèse herméneutique qui guidera notre propos: en réactivant la période d'élaboration de Sein und Zeit, ainsi que l'aristotélisme phénoménologique et existential de Heidegger appliqué au «corps vivant»(Leib), il devient possible de recomposer ou de rafraîchir la mémoire de Heidegger, si l'on peut dire, voire de la compléter par certaines greffes ou prothèses là où elle faillit, pour lui faire dire ce qu'elle se refuse à dire, puisque dans l'entretien avec $\mathrm{M}$. Boss du 3 mars 1972, Heidegger ne paraît pas se défendre contre le reproche bien connu de Sartre, selon lequel il n'aurait écrit que six lignes sur le Leib (Sartre dit évidemment «corps»): «Je ne puis répondre à l'objection de Sartre qu'en constatant que ce qui concerne

\footnotetext{
${ }^{5}$ Heidegger, Zollikoner Seminare, p. 116-117. Sous la référence vague à une certaine Leibesphänomenologie française, fatalement latinisée, il faut sans doute deviner les noms de Sartre, de Waehlens et Merleau-Ponty ; voir sur ce point Richard Askay, "Heidegger, the body and the french philosophers ", in Continental philosophy review, 1999, 32 (1), p. 29-35. Comme opérateur historique de cette latinisation du sôma grec, Heidegger songe peut-être tout particulièrement au commentaire du De anima par Thomas d'Aquin, où sôma est traduit par corpus.

${ }^{6}$ Heidegger, Zollikoner Seminare, p. 116-117.
} 
le corps vivant (das Leibliche) est le plus difficile, et qu'à l'époque, je $\mathrm{n}^{\prime}$ ai rien su en dire de plus $»^{7}$.

Plutôt que de nous engager dans une reconstruction de la problématique du Leib par une interprétation immanente des Séminaires de Zollikon, nous partirons ainsi de certaines réminiscences heideggériennes qui nous ramènent à la période d'élaboration de Sein und Zeit. Cette incursion vers une version antérieure du programme heideggérien qu'est l'élaboration, par le biais d'une Destruktion phénoménologique d'Aristote, d'une problématique du Leib dès les années 1920, devrait permettre d'éclairer en retour le traitement du Leib dans les Séminaires de Zollikon et d'en opérer, sur le mode d'une remémoration, une mise à jour pour en complexifier la donne.

Ce que cette destruction phénoménologique de la définition aristotélicienne implique et met en jeu, c'est, ni plus ni moins, la destruction critique du "psycho-somatique », c'est-à-dire de la distinction psuchè et sôma, récurrente dans les Séminaires de Zollikon. Or c'est précisément le concept existential de Leib, procédant de cette destruction critique du psycho-somatique, qui est censé procurer le fondement à toute "médecine psycho-somatique», comme Heidegger l'indique lors de son entretien avec M. Boss du 3 mars 1972 ${ }^{8}$, c'est-àdire, également, à une certaine Daseinsanalyse qui se réclamerait de l'analytique du Dasein de Sein und Zeit revue et corrigée par les Séminaires de Zollikon.

Le réquisit premier pour faire émerger la configuration phénoménologique du Leib, c'est, Heidegger le rappelle dans les entretiens siciliens avec M. Boss au printemps 1963, son inscription dans l'êtreau-monde. Le phénomène du Leib présuppose une élaboration de l'être-au-monde existential :

1. Le traitement des phénomènes du Leib n'est aucunement possible sans une élaboration suffisante des traits fondamentaux de l'être-au-monde existential. 2. Il n'y a pas encore de description suffisamment utilisable du phénomène de la chair, c'est-à-dire une description telle qu'elle soit vue depuis l'être-au-monde 9 .

\footnotetext{
${ }^{7}$ Ibid., p. 292. À une objection similaire avancée par Hans Jonas, Heidegger aurait répondu : "Oui, je l'ai toujours oublié » (H. Blumenberg, Die Verführbarkeit des Philosophen, Francfort-surle-Main, Suhrkamp, 2000, p. 64). L'objection sartrienne, déjà formulée dès la parution de Sein und Zeit par Löwith et Plessner, est régulièrement rééditée ; cf. par exemple, dans les années 1980, Didier Franck, Heidegger et le problème de l'espace, Paris, Minuit, 1986, p. 126 : «Sein und Zeit échoue sur le problème de la chair ». Cf. plus généralement Jean Greisch, "Le phénomène de la chair: Un "ratage" de Sein und Zeit ", in G. Floviral (éd.), Dimensions de l'exister. Etudes d'anthropologie philosophique, Louvain, Peeters, 1994, p. 154-177.

${ }^{8}$ Heidegger, Zollikoner Seminare, p. 296.

${ }_{9}^{9}$ Ibid., p. 202.
} 
Or, cette description "utilisable » existe, au moins virtuellement, dans le cours marbourgeois de 1924 sur Les concepts fondamentaux de la philosophie aristotélicienne, où Heidegger évoque, à partir du De anima, l'être du vivant dans son monde et, plus précisément, dans son pathos ou dans sa Befindlichkeit. Une Befindlichkeit qui fait d'ailleurs une brève apparition dans les Séminaires de Zollikon, où Heidegger note, de façon elliptique : "La Befindlichkeit fonde le se trouver-bien ou le se trouver-mal selon les circonstances (das jeweilige Wohl- und Missbefinden), mais elle est fondée à son tour par l'être-exposé (Ausgesetztheit) de l'homme à l'étant dans sa totalité ${ }^{10}$.

Pour mettre en perspective le concept de Befindlichkeit à partir de sa matrice aristotélicienne, il faudrait gloser notamment le terme de "disposition" (diathesis) dans Mét. 1022b1-3 : " "Disposition" s'entend de l'ordre de ce qui a des parties, selon le lieu, la puissance ou la forme. Il faut bien, en effet, qu'il y ait là une certaine position, comme l'indique le mot même de "disposition" ». Mais, dans le cours de 1924, Heidegger obtient le sens principal de Befindlichkeit par une traduction interprétative de kataphasis et diathesis dans Rhét. 1369b33 1370a2 :

\begin{abstract}
Admettons comme postulat que le plaisir est un mouvement de l'âme

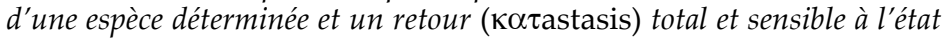
naturel, et que la peine est le contraire. Si c'est bien en cela que consiste le plaisir, il est clair aussi que ce qui produit la disposition temporaire (סtathesis) susdite est agréable et que ce qui la détruit ou produit l'état contraire est pénible ${ }^{11}$.
\end{abstract}

Venons-en directement à ce qui, à partir de ce passage, permet à Heidegger d'élaborer le rapport phénoménal particulier dans la définition de la Befindlichkeit. Lorsque le plaisant (hèdu), " ce qui élève » (das Hebende), arrive et se présente (begegnet) au vivant, celui-ci se trouve déjà dans un monde :

Une disposition déterminée est là d'avance, en sorte que la production d'une disposition déterminée par le plaisant signifie qu'une disposition, affectée (angegangen) par le plaisant, se transporte (sich versetzt), en se disposant, dans une nouvelle disposition (déterminée par le nedù -

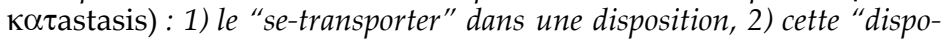
sition" même dans laquelle on est porté12.

\footnotetext{
${ }^{10} \mathrm{Ibid} .$, p. 182.

${ }^{11}$ Heidegger, Grundbegriffe der aristotelischen Philosophie (1924), GA 18, p. 48.

12 Ibid., p. 54.
} 
Heidegger reconduit cette double transposition (doppelte Übersetzung) au caractère actif et passif qui marque tous les phénomènes fondamentaux de la vie: "Tous les modes de la vie, du vivre, sont caractérisés par le fait que la manière de l'être est alors une disposition sur le mode d'un (trans)porter-dans-une-disposition-et-y-être (In-eine-Befindlichkeit-Bringen-und-darin-Sein) $»^{13}$.

Le vivant humain n'est proprement disposé qu'en se situant dans cette disposition : c'est en se réjouissant qu'il se transporte dans la joie. Toute disposition est affectivement déterminée (bestimmt) par une Stimmung, comme le dira Heidegger dans Sein und Zeit, selon ce qu'il appelle, dans les Séminaires de Zollikon, un «se trouver-bien ou se trouver-mal selon les circonstances ».

S'il est vrai que le Leib est le lieu matériel ou le site des affects, leur matière, la configuration conceptuelle de la Befindlichkeit permet de circonscrire le champ où pourrait s'inscrire le problème du Leib, parce que la Befindlichkeit, et son exposant ou indice qu'est la Stimmung, l'état affectif, si nous pouvons ainsi sous-traduire le terme de Stimmung, impliquent d'emblée une dimension spatiale, un site («naturel »), que serait le Leib comme « espace», ou " ouverture » du vivant.

Dans son cours de 1924, Heidegger souligne en effet que les pathè (les affects ou affections de l'âme) ne sont pas des états psychiques, car «il s'agit d'une disposition du vivant (Befindlichkeit des Lebenden) dans son monde, selon la manière qu'il a d'être disposé (gestellt) à l'égard d'une chose, de se laisser affecter par elle ${ }^{14}$. Heidegger précise :

Les pathè sont des traits caractéristiques qui déterminent plus précisément la manière d'être-au-monde (In-der-Welt-Sein) [...]. Il ne s'agit pas d'états psychiques" avec des "symptômes somatiques", car les pathè caractérisent l'homme entier dans sa disposition affective dans le monde (Befindlichkeit in der Welt). L'homme entier (das Ganze des Menschen) est l'objet premier dont traite la psychologie aristotélicienne dans le De anima, livre I. Eu égard à son être, le tout de l'homme doit être compris comme zôè, comme être-au-monde - selon cette conception, il ne s'agit pas à proprement parler d'une thématisation pour la psychologie, mais d'une exposition de l'être de cet étant ${ }^{15}$.

Lorsque Heidegger parle de $\mathrm{l}^{\prime}$ « homme entier » objet de la psychologie aristotélicienne, il fait plus exactement allusion à De an. 408b115: « Nous disons en effet de l'âme qu'elle est triste ou joyeuse,

\footnotetext{
${ }_{13}$ Ibid., p. 54.

${ }^{14}$ Ibid., p. 122.

${ }^{15}$ Ibid., p. 192 ; ibid., p. 122 : «Les pathè, les affects, ne sont pas des états psychiques ; il s'agit d'un être-diposé $d u$ vivant (Befindlichkeit des Lebenden) dans son monde, selon la manière qu'il a d'être disposé à l'égard d'une chose, de se laisser affecter par elle ».
} 
audacieuse ou craintive, et aussi irascible, sensitive, pensante » ; mais, corrige Aristote plus loin, « dire que l'âme est en colère, c'est comme si l'on prétendait que c'est l'âme qui tisse ou qui construit. Il est sans doute préférable, en effet, de ne pas dire que l'âme éprouve de la pitié, apprend ou pense, et de dire que c'est l'homme, par son âme»; c'est-à-dire non pas l'âme elle-même mais le « sujet » comme vivant animé.

Il faut rappeler ici le statut spécial pour Heidegger du De anima qu'il ne lit pas comme une psychologie, mais comme une " ontologie de la vie »; et selon Heidegger, donc, ne pas avoir vu qu'il s'agissait d'une ontologie du vivant dans le monde est le "péché » premier de la scolastique, mais aussi de toute la tradition moderne, Kant compris, qui critique la notion de Seelensubstanz dans les Paralogismes. À le soumettre à une lecture strictement phénoménologique qui mettrait entre parenthèses toutes les strates du commentaire scolastique déposées sur les phénomènes initialement mis en évidence par Aristote, le Traité de l'âme se présente ainsi comme une ontologie des degrés du vivant (Stufen des Lebendigen) végétal, animal et humain, le livre III traitant spécifiquement du vivant humain dans le monde et de son $\log _{0 S^{16}}$.

Heidegger, dans les Séminaires de Zollikon où il entend "déconstruire » le psycho-somatique comme base de toute médecine psychosomatique, mais aussi de toute Daseinsanalyse future, se souvient quelque peu de son propre laboratoire conceptuel des années 1920, on l'a vu, lorsqu'il traduit la définition canonique de l'âme par «la psyché est le mode d'être d'un vivant»; définition qui d'ailleurs apparaissait non seulement au gré des cours sur Aristote, mais aussi dans Sein und Zeit, sous une forme condensée, lorsque Heidegger notait que la vie est une "manière spécifique d'être " (eigene Seinsart $)^{17}$. Toujours est-il que pour lire le De anima comme une ontologie phénoménologique de la vie, et plus particulièrement de la vie humaine, le Heidegger aristotélisant des années 1920 opère, en la radicalisant, une connexion essentielle entre la vie et l'être ${ }^{18}$.

\footnotetext{
${ }^{16}$ Cf. Heidegger, Die Grundbegriffe der Metaphysik (1929-30), GA 29/30, p. 454 ; Einführung in die phänomenologische Forschung (1923-24), GA 17, p. 6 et p. 293.

${ }_{17}$ Heidegger, Sein und Zeit, Tübingen, Niemeyer, 2001, p. 50 ; Aristoteles, Metaphysik IX 1-3. Von Wesen und Wirklichkeit der Kraft (1931), GA 33, p. 120 : "Or la "vie" est une manière de l'être ».

${ }^{18}$ Connexion légitime, si l'on considère que "animé » (empsuchon, besselt), est en quelque sorte synonyme d' «être en vie ", de vivant (zèn, De an., 413b20 sq.), et surtout si on se souvient qu'il y a chez Aristote, dans le De an. et ailleurs, une sorte d' «équivalence » entre "vivre » (zèn) et " être » (einai), équivalence qui vaut pour tout vivant, selon De an. II, 4, 415b10-15: «Vivre (zèn) est, pour les vivants, leur être (einai) même, et la cause et le principe de ceux-ci, c'est l'âme ». On trouve une brève traduction paraphrastique de ce passage crucial dans GA 22 (1926), p. 184 :
} 
Après ces quelques précisions sur le statut singulier, ontologique, du De anima pour Heidegger, revenons au cours de 1924 et à la problématique du vivant dans le monde comme disposition affective. Intéressons-nous $\mathrm{d}$ 'abord aux traits principaux de la lecture heideggérienne de la doctrine aristotélicienne du pathos. La question générale sous l'horizon de laquelle Aristote traite des affects ou affections dans le De anima est selon Heidegger celle-ci : "Comment quelque chose peut-il arriver (geschehen) à un vivant eu égard à son être ? ${ }^{19}$, entendu que l'être d'un étant vivant est sa psuchè.

La destruction heideggérienne du concept de pathos doit être lue à la lumière de sa matrice qu'est la théorie aristotélicienne des affects et de la crainte dans la Rhétorique, lecture que nous ne pouvons entreprendre ici. D'autre part, pour exposer l'interprétation heideggérienne du Pathos, il faudrait retracer l'analyse heideggérienne des quatre sens du pathos selon Mét. IV, 21 ; contentons-nous ici d'indiquer le résultat phénoménologique de ces analyses.

Des quatre sens du pathos, Heidegger dégage en effet une référence essentielle, à savoir que le pathos est " référé à l'être du vivant (Sein des Lebenden) caractérisé par un être-disposé-ainsi-dans-telle-ou-telle-circonstance (Je-und-je-sich-so-Befinden) $»^{20}$. Après avoir synthétisé les quatre sens de pathos en les référant au vivant, Heidegger, au $\S 18 \mathrm{c}$ ), souligne le caractère intégral du pathos référé au vivant humain, ou à l'existant humain, qui vit dans le logos, qui possède le logos, caractère distinctif de l'homme par rapport au vivant animé privé de logos. Ainsi, le

pathos [référé à la zồ̀ praktikè meta logou] est donc un être-emporté (Mitgenommenwerden) de l'être-là. Il est emporté par cela même qui est là avec lui dans le monde - par le dehors, mais par le dehors en tant que monde, lequel est le ce-dans-quoi (Worin) de mon être. C'est de l'être-là lui-même que procèdent les possibilités et les manières de son êtreemporté $e^{21}$.

Ce pathos comme être-emporté, cet être-décontenancé (Aus-derFassung-Sein) de l'être-là, par exemple dans la colère, n'est pas un phénomène affectif localisé dans un psychisme, manifesté par des épiphénomènes qui seraient autant de symptômes somatiques : c'est

\footnotetext{
« L'âme n'est pas un étant (le psychique) à côté d'un corporel (physique), mais la manière d'être d'un corporel (ein Körperliches) déterminé, en sorte que celui-ci, en raison de cet être comme vivant (das Lebende), se distingue de l'inanimé (das Leblose) ».

${ }^{19}$ Heidegger, GA 18 (1924), p. 200.

${ }^{20}$ Ibid., p. $195-196$.

${ }^{21}$ Ibid., p. 197.
} 
toujours, il faut y insister, l'intégralité du vivant dans le pathos comme être-au-monde corporel et vivant (leibliches In-der-Welt-sein) qui se trouve emporté22.

Toujours au $\S 18 \mathrm{c}$ ), Heidegger précise cette disposition affective du vivant par rapport au sôma, au Leib, en sollicitant les notions de phantasia et de nô̂s. Souvenons-nous de ce que notait Heidegger dans l'entretien sicilien de 1963 avec M. Boss : «Il n'y a pas encore de description suffisamment utilisable du phénomène du Leib, c'est-àdire une description telle qu'elle soit vue depuis l'être-au-monde $»^{23}$.

Or, quelques éléments de cette " description suffisamment utilisable " se trouvent, selon nous, dans ces passages du De anima commentés par Heidegger, passages où Aristote montre que «le noûs de l'être humain se rapporte à la phantasia et par-là à l'aisthèsis et au paskhein du sôma ${ }^{24}$.

C'est dans ces passages que Heidegger a repéré dès 1924 l'esquisse d'une configuration que seule la phénoménologie, dit-il dans le cours, aurait timidement commencé à exploiter, en récusant toute séparation des actes psychiques et des actes corporels ${ }^{25}$. Il y a bien une distinction de la psuchè et du sôma, mais ces deux versants d'un même phénomène sont synthétisés dans le corps vivant animé, c'est-à-dire dans le Leib, synthèse psycho-somatique que Heidegger, dans Sein und Zeit, pensera sous le terme technique d'Existenz.

$\mathrm{Au} \S 18 \mathrm{c})$, Heidegger discute donc De an. 403a3-10 :

Une difficulté se présente aussi à propos des affections de l'âme : sont-elles toutes communes à l'être qui possède l'âme ou bien y en a-t-il aussi quelqu'une qui soit propre à l'âme elle-même? Le déterminer est indispensable, mais difficile. Il apparaît que, dans la plupart des cas, il n'est aucune affection que l'âme puisse, sans le corps, subir ou exercer : telle la colère, l'audace, l'appétit et, en général, la sensation. S'il est pourtant une opération qui semble par excellence propre à l'âme, c'est l'acte de penser; mais si cet acte est, lui aussi, une espèce d'imagination ou qu'il ne puisse exister indépendamment de l'imagination, il ne pourra pas davantage exister sans un corps.

Ainsi, c'est au stade de cette interprétation active de De an. 403a310 en 1924 qu'il est possible de considérer que Heidegger, compte tenu de la source ou ressource aristotélicienne constitutive de la conceptualité de Sein und Zeit, n'a aucunement négligé le phénomène

\footnotetext{
22 Ibid., p. 197-208, sur Leib et Leiblichkeit.

${ }^{23}$ Heidegger, Zollikoner Seminare, p. 202.

${ }^{24}$ Heidegger, GA 18 (1924), p. 202.

${ }^{25}$ Ibid., p. 199.
} 
du sôma, ou du Leib, comme " matière » des affects ; l'être-au-monde, comme être-affecté "naturel », est toujours déjà aussi un être corporellement vivant, un «être-dans-le-corps-vivant». La périphrase que Heidegger propose pour sôma à la fin du passage cité, où Aristote dit que l'acte de penser, étant une espèce d'imagination, ou représentation (phantasia), ne saurait exister sans corps vivant, est à cet égard pour le moins instructive: "la vie intégrale de l'être humain (das ganze Leben des Menschen) ${ }^{26}$.

Pour autant que le vivant humain est affecté par le monde, qu'il est sujet aux pathèmes (pathemata, Geschehnisse) qui lui adviennent du monde dans lequel il se trouve toujours déjà, il est simultanément affecté dans son Leib conçu comme pleine existence (Dasein) du vivant ${ }^{27}$, c'est-à-dire que les affects, générés par les pathèmes externes et circonstanciels, supposent comme compagnon le Leib, mon Leib, lequel participe donc de la genèse des affects de la psuchè. L'affect est ainsi donné en même temps que le Leib. Aucun affect ne saurait être séparé d'un Leib situé dans le monde, ni aucune conduite intentionnelle isolée de l'intégralité de l'être humain comme être vivant ; certains affects d'ailleurs peuvent être générés par le Leib sans aucune cause externe, comme l'affect de la crainte, selon l'exemple d'Aristote.

Dans ce sens, les affects ou affections de la psuchè sont, comme dit Aristote, des « raisons matérielles», ou des «formes inhérentes à la matière » (De an. 403a24). Les affections de l'âme ne sont pas séparables de la matière physique des animaux, des vivants animés, ce qui implique par ailleurs de soumettre les affects à une investigation "physique», en étudiant dès lors le mouvement, problématique importante que nous n'aborderons pas ici.

La matière (hulè) des affects, la matière susceptible de rendre possible la vie et l'être-au-monde des vivants animés, c'est donc le $L_{e i b}{ }^{28}$. Le Leib (sôma), n'est pas pour autant synonyme de matière (hulè) ; car le Leib, comme corps vivant animé, doit être conçu, Heidegger souligne ce point qui selon lui n'est pas saisi par Aristote, comme matière physique au sens d'un pouvoir (Können), d'une possibilisation (das Vermögende), dont l'actualisation ou l'entéléchie (la forme) est l'âme (psuchè).

La main n'est que main pour autant qu'elle est vivante et animée, capable de saisir ou de sentir, c'est-à-dire pour autant qu'elle est

\footnotetext{
${ }^{26}$ Ibid., p. 199.

${ }^{27}$ Ibid., p. 202.

${ }^{28}$ Ibid., p. 203 : «Leur hulè n'est rien d'autre que le sôma, la corporéité vivante (Leiblichkeit) de l'homme "; ibid., p. 204 : « sôma est une hulè déterminée, dont la caractéristique consiste à rendre possible la vie ».
} 
organisée par le pouvoir de la psuchè; ou encore, selon l'exemple récurrent chez Heidegger, qu'il cite aussi dans les Séminaires de Zollikon, c'est parce que nous avons le pouvoir de voir que nous avons des yeux physiques, et non l'inverse ${ }^{29}$. C'est aussi pourquoi Heidegger soulignera plus généralement dans le Séminaire de Zollikon du 3 mars 1972, fidèle à l'aristotélisme de ses débuts, que le Leib ressort à l'existence, et non à la matière inanimée (leblose Materie), et c'est aussi pourquoi il pourra y dire que le Leib relève du domaine invisible, c'est-à-dire in-commensurable, d'une perception, plus précisément d'un pouvoir de saisir les significativités (Vernehmen-können der Bedeutsamkeiten) de ce qui arrive et de ce qui fait encontre (das Begegnende), à la lumière (Lichtung, Gelichtetheit) d'une certaine ouverture au monde (Welt-Offenständigkeit) ${ }^{30}$.

Cette question de la matière et du pouvoir psychique nous conduit, on l'aura deviné, au problème capital de l'intellect ou intelligence (nô̂s). Insistons sur le lien controversé, décisif pour notre problématique de la disposition affective du Leib, entre phantasia et noèsis selon De an. 403a3-10. La particularité de l'acte de penser, c'est qu'il n'est pas limité à un champ sensoriel particulier, mais qu'il vise, sur un mode universalisant, tous les étants possibles (ta panta), quand bien même ceux-ci seraient absents. Sans en approfondir la notion selon la reprise phénoménologique par Heidegger (qu'on retrouve notamment dans la critique de la Bildwahrnehmung et de la Leibhaftigkeit chez Husserl dans son cours de $1925^{31}$ ), retenons que la phantasia est ici un "présentifier» (Vergegenwärtigen) du monde, où la chose représentée $n^{\prime}$ est pas nécessairement présente ${ }^{32}$ : la phantasia est une re-présentation au sens d'une "répétition de ce qui a été présent »33, selon une présentification (Vergegenwärtigung) qui se re-présente l'objet absent qu'est la gare de Zurich, pour citer l'exemple des Séminaires de Zollikon ${ }^{34}$.

«Jamais (donc) l'âme ne pense sans image»(De an. 431a15), ou plutôt sans représentation : ce n'est pas quelque processus cérébral, mais l'acte d'imaginer, de re-présenter, avec la dimension aisthétique, et donc corporelle et vivante, qu'il implique, qui est au fondement de

\footnotetext{
${ }^{29}$ Ibid., p. 233 ; Zollikoner Seminare, p. 293.

${ }^{30}$ Ibid., p. 292-293, ce qui rejoint ce qu'il disait dès le premier séminaire au Burghölzli de Zurich, le 8 septembre 1959 (ibid., p. 4).

${ }^{31}$ Heidegger, Prolegomena zur Geschichte des Zeitbegriffs, GA 20 (1925), p. 52-58.

32 Heidegger, GA 18 (1924), p. 199.

${ }^{33}$ Ibid., p. 202.

${ }^{34}$ Heidegger, Zollikoner Seminare, p. 86-96, p. 110.
} 
l'acte de penser. C'est ce que semble dire Heidegger lorsqu'il précise, toujours dans le cours de 1924 :

Dans la mesure où la noèsis est la possibilité suprême pour l'être de l'homme, l'intégralité de l'être humain est déterminé en sorte qu'il doit être saisi comme l'être-au-monde corporel et vivant de l'homme (das leibmässige In-der-Welt-sein des Menschen) ${ }^{35}$.

Autrement formulé : la Leiblichkeit, comme corporéité vivante, est le fondement premier de l'être humain, du Dasein ${ }^{36}$. Or, dans cette configuration noûs - phantasia - aisthèsis - sôma, où s'inscrit la disposition affective du vivant dans son Leib, le rôle ambigu de l'intellect (noûs) convient d'être précisé et discuté, car sa dépendance au corps vivant n'est pas évidente, la position de Heidegger étant d'ailleurs fluctuante sur ce point. Alors qu'Aristote affirme dans le De anima que «l'âme n'est pas séparable du sôma» (413a4), simple conséquence de ce que l'âme est considérée comme la réalisation première, l'entéléchie du sôma, l'intellect théorétique pourtant est, dit-il, «un genre de l'âme tout différent» et il se peut que «lui seul puisse être séparé du sôma, comme l'éternel, du périssable»(413b24-25). Nous reviendrons sur ce problème, traditionnel, de la séparabilité ou de la non-séparabilité de l'âme plus loin ; en attendant, concentrons-nous sur un aspect important du noûs, qui est son caractère d'ouverture et de virtualité.

Au livre III, Aristote définit le noûs en le comparant à la lumière : "C'est en quelque sorte un état analogue à la lumière (phos); car, en un certain sens, la lumière, elle aussi, convertit les couleurs en puissance, en couleurs en acte. Et c'est cet intellect qui est séparé, impassible et sans mélange, étant par essence un acte » (430a15-19). Le noûs est analogue à la lumière qui actualise les couleurs existant en puissance dans l'obscurité.

Dans son commentaire de 1924, Heidegger reprend la métaphore de la lumière rapportée au noûs, tout en paraphrasant le passage : de même que chaque couleur ne parvient à son existence, au «là » de son être-là, que par la lumière, de même tout étant qui est là nécessite une mise en lumière (Erhellung) fondamentale pour être là ${ }^{37}$. Et cet étant qui est là doit avoir par lui-même la possibilité d'être ouvert (Aufgeschlossensein), cette possibilité étant précisément le noûs, fonc-

\footnotetext{
35 Heidegger, GA 18 (1924), p. 199.

${ }^{36}$ Ibid., p. 199.

${ }^{37} \mathrm{Ibid} .$, p. 200-201. Heidegger résume ici son commentaire de la doctrine aristotélicienne de la lumière et des couleurs dans GA 17 (1923-24), p. 6-13, commentaire qu'il exploitera dans le célèbre $\S 7$ de Sein und Zeit.
} 
tion du nô̂s que nous retrouvons comme ouverture noétique primordiale (Lichtung) et être-ouvert (Erschlossenheit) dans Sein und Zeit.

$\mathrm{Au} \S 28$ de Sein und Zeit, Heidegger note en effet, selon une référence implicite au passage du De anima cité plus haut, que le Dasein est « en lui-même éclairé (gelichtet) comme être-au-monde, non pas par un autre étant, mais en sorte qu'il est lui-même l'éclaircie (die Lichtung). Ce n'est qu'à un étant existentialement éclairé de la sorte que l'étant subsistant devient accessible à la lumière, ou demeure caché dans l'obscurité $»^{38}$.

Revenons à la description du noûs à partir de la lecture heideggérienne du De anima en 1924. Le nô̂s, comme "en puissance» (dunaton, 429a22), est défini comme pouvoir de saisir et percevoir (Vernehmen) l'aspect ou l'idée (eidos) des choses pour les mettre en lumière et permettre de les voir. Heidegger résume : "La détermination fondamentale du noûs, de la "visée intentionnelle" de quelque chose ("Vermeinen" von etwas), c'est le dunaton, la possibilité pure et simple de l'être-ouvert (Aufgeschlossensein), du là $(D a)$ de quelque chose - toute saisie concrète se meut et se tient dans cet êtreouvert $»^{39}$.

On peut remarquer ici, en passant, à propos de la traduction de noûs par Vermeinen, que c'est à partir d'une réinterprétation du nô̂s aristotélicien enraciné dans le Leib que Heidegger a articulé un aspect fondamental de sa critique de Husserl, notamment de la structure de l'intentionnalité déterminée par la distinction noèse/noème, Heidegger reprochant à Husserl d'opérer une surdétermination théorique de cette structure primitive de l'intentionnalité, structure qui se donne, à réinterpréter Aristote, comme un tendre-vers (Sich-richten-auf) toujours déjà corporel et affectif ${ }^{40}$.

Autre remarque, c'est à partir de cette ouverture noétique préthéorique fondamentalement ancrée dans le Leib, que pourrait se poser le problème de la spatialité du vivant ou de son espacement (Räumen, Einräumen). C'est peut-être d'ailleurs aux analyses du De anima de 1924 que Heidegger fait allusion dans Sein und Zeit lorsqu'il note que la spatialisation (Verräumlichung) du Dasein dans sa corporéité vivante (Leiblichkeit), abrite une problématique qui ne saurait être traitée dans le cadre de Sein und Zeit ${ }^{41}$. La spatialité fait également question, on le sait, dans les Séminaires de Zollikon, par exemple

\footnotetext{
${ }^{38}$ Heidegger, Sein und Zeit (1927), p. 133.

${ }^{39}$ Heidegger, GA 18 (1924), p. 201.

${ }^{40}$ Heidegger, GA 20 (1925), p. 61. Cf. Zollikoner Seminare, p. 261.

${ }^{41}$ Heidegger, Sein und Zeit (1927), p. 108.
} 
lorsque Heidegger parle de l'«être-orienté essentiel (wesensmässige Ausrichtung) de notre Da-sein » et qu'il affirme que "le Dasein n'est pas spatial parce qu'il est corporel et vivant (leiblich), mais la corporéité vivante (Leiblichkeit) n'est possible que parce que le Dasein est spatial au sens où il est spatialisant (einräumend) $»^{42}$.

La nature du noûs est donc d'être en puissance, mais en tant que virtualité, le noûs est en lui-même « impassible »; Aristote note en De an. 429a15-17 : "Il faut donc que cette partie de l'âme soit impassible, tout en étant susceptible de recevoir la forme ; qu'elle soit, en puissance, telle que la forme, sans pour autant être cette forme ellemême ».

Or, Heidegger réinterprète ce principe impassible qu'est le noûs selon Aristote comme un caractère transcendantal et "méta-humain », le noûs pouvant saisir universellement tous les étants au-delà de la sphère simplement humaine: le noûs, ne pouvant être lui-même affecté, car il est « en puissance » (il est un Seinkönnen, comme dira Sein und Zeit), est comme la condition de possibilité pour que le vivant, en général, puisse être affecté "en acte» par quelque chose dans le monde. Pour le dire avec Heidegger: "Le noûs est la condition de possibilité fondamentale de l'être-au-monde, laquelle condition, en tant que telle, dépasse l'être concret et singulier de l'homme individuel $\gg^{43}$.

$C^{\prime}$ est en s'autorisant de cette constitution transcendantale, qui ouvre sur la possibilité de l'activité théorétique comme vie proprement philosophique capable de saisir l'être, que Heidegger distingue le corps animal du Leib humain ${ }^{44}$, distinction susceptible d'éclairer l'ajout de Heidegger, dans les Séminaires de Zollikon, à la définition canonique de l'âme, citée au début, à savoir « l'entéléchie de l'homme est le $\log o s »$. Cette distinction heideggérienne, qui sur ce point prolonge et radicalise Aristote, entre corps animal et Leib humain est réglée par la distinction entre zôè et bios, c'est-à-dire par la distinction entre « vie » et « existence $»^{45}$.

L'entéléchie de l'être humain, c'est-à-dire cette sorte d'âme qu'est le noûs comme entéléchie du corps vivant humain, c'est donc le logos.

\footnotetext{
42 Heidegger, Zollikoner Seminare, p. 293-294 et p. 105.

${ }^{43}$ Heidegger, GA 18 (1924), p. 201.

${ }^{44}$ Distinction récurrente dans l'œuvre reconduisant à la destruction critique du De anima opérée dans les années 1920 ; cf. par ex. Lettre sur l'humanisme (1946), in GA 9, p. 324 : « Le Leib de l'être humain est quelque chose d'essentiellement différent d'un organisme animal »; Heidegger/Fink, Heraklit (1966-67), p. 237 : «L'élément corporel et vivant (das Leibliche) en l'homme n'est pas quelque chose d'animal ».

${ }^{45}$ Sur ce point, qu'il nous soit permis de renvoyer à notre étude « Métaphysique du vivant. Note sur la différence zoo-anthropologique de Plessner à Heidegger », in Philosophie, no 116 (2012), p. $48-77$.
} 
L'animalité, par le truchement humain du logos, constitue pour ainsi dire le «bio-logique»; la vie humaine n'est jamais seulement zôè, mais aussi bios ${ }^{46}$. Les plantes et les animaux se définissent comme vie au sens de zôè, mais ils sont dépourvus de bios, ils n'ont pas de vie au sens, comme le dit Heidegger en 1924, de l'histoire d'une vie (Lebensgeschichte, « biographie ») :

()ios: un nouveau concept de "vie", à ne pas confondre avec zôè. La biologie moderne ne se réfere justement pas au bios grec. ๑ios veut dire "moyens de vie", "chemin de la vie", la temporalité spécifique d'une vie de la naissance à la mort, "le parcours d'une vie", en sorte que oios signifie également "récit d'une vie": le comment d'une zôè est le ๑ios, l'histoire d'une vie ${ }^{47}$.

En raison du logos, le corps vivant humain n'est pas le corps vivant animal: "L'homme se distingue de l'animal parce qu'il peut en général "dire" (sagen), c'est-à-dire qu'il possède le langage (Sprache) » ${ }^{48}$. Pour l'existant humain, la vie ne se réduit pas à la vie "zoologique » et à ses mouvements primordiaux (nutrition, croissance, dépérissement), lesquels sont pour ainsi dire relevés (aufgehoben) par le logos comme possibilité d'être conductrice ou hégémonique (führende Seinsmöglichkeit) ${ }^{49}$. C'est aussi ce qui explique que, sur un mode performatif, l'investigation qui porte sur le Leib se fait selon une certaine idée régulatrice, une certaine perspective qui n'est autre que celle du corps vivant animé doué de logos et performant l'acte philosophique, plus précisément depuis une activité théorétique, qui est alors comme en position d'excentricité par rapport à la centralité du corps vivant animal que l'être humain est aussi, d'une certaine façon, mais auquel il ne peut jamais accéder que de façon indirecte ou privative, c'est-àdire logique :

Il s'agit de mettre en évidence l'être-là excellent (das eigentliche Dasein), afin d'examiner si possible le "physiologique", les "états du Leib". Ainsi, dans la considération du somatique, l'orientation est donnée par l'eidos de la vie humaine, caractérisée comme zôè praktikè metà logou ${ }^{50}$.

\footnotetext{
${ }^{46}$ Aristote, Eth. Nic., I, 6, 1097b33 : « Le simple fait de vivre est, de toute évidence, une chose que l'homme partage en commun même avec les végétaux; or ce que nous cherchons, c'est ce qui est propre (idion) à l'homme». Cf. la paraphrase en GA 22 (1926), p. 312 : «Quel est le bios le plus haut, la possibilité la plus haute de l'existence, la manière d'être par laquelle l'homme suffit au plus haut degré à son pouvoir d'être qui lui est spécifique, par laquelle l'homme est proprement (eigentlich) lui-même? ".

47 Heidegger, GA 18 (1924), p. 74 ; GA 33 (1931), p. 123.

${ }^{48}$ Heidegger, Zollikoner Seminare, p. 114.

${ }^{49}$ Heidegger, GA 17 (1923-24), p. 296.

50 Heidegger, GA 18 (1924), p. 232.
} 
Dans la vie naturelle même, qui n'est accessible que par voie indirecte, se pose la question du bien véritable, propre à l'être humain : la question du «bien-vivre » (eu zèn) et d'une "vie heureuse » (euzôia $)^{51}$. Quel est dès lors le type de vie, le bios suprême, qui permet à la zồ humaine d'accéder au bonheur parfait, à l'Eigentlichkeit de l'existence, une Eigentlichkeit, ou excellence propre, qui serait alors comme «la vie de la vie ", la vie au carré, distincte de la vie simplement animale? On connaît la réponse d'Aristote : c'est le bios theôrètikos dont l'excellence surpasse la « vie de jouissance » et la « vie politique » ${ }^{52}$.

La réponse de Heidegger à cette question décisive, laquelle réponse procède d'une destruction critique du primat aristotélicien de la vie théorétique dans les années 1920 et dans Sein und Zeit, serait peut-être que ce type de vie est certes l'activité théorétique, mais que cette activité théorétique est toujours située dans le monde, est toujours incarnée et donc essentiellement finie.

Dans la controverse traditionnelle sur l'inséparabilité ou nonséparabilité de l'intellect agent à l'égard du corps vivant, où se détermine le rapport entre corps et âme, Heidegger donc pencherait, a penché, pour la première solution. Car s'il reprend dans Sein und Zeit, on l'a vu brièvement, la fonction du noûs comme ouverture noétique primordiale (Lichtung) et donc comme vérité, sa reprise tend à contester toute séparabilité, consolidant les leçons de la dépendance du noûs à l'égard de la phantasia et donc du corps vivant dans le De anima $^{53}$, c'est-à-dire en soulignant l'irréductibilité de l'être-au-monde. Cette dépendance se retrouve d'ailleurs dans le cours de 1929-30, où Heidegger parlera de cette vérité pré-logique, anté-prédicative, où s'opère la Weltbildung, autrement dit l'imagination transcendantale kantienne interprétée par lui, à la même époque, comme marque de la finitude humaine.

Dans Sein und Zeit, le concept de vie philosophique incarnée, c'està-dire le concept de Dasein en son excellence propre, ou Eigentlichkeit, qu'est le mode de la vorlaufende Entschlossenheit comme puissance logique (dunamis meta logou) radicalement finie, mais capable d'anticiper le futur, est l'un des aboutissements de la destruction heideggérienne du "psycho-somatique » opérée à partir du De anima dès 1924, schème " psycho-somatique » dont Heidegger rappelera dans les Séminaires de Zollikon qu'il est au fondement d'une conception métaphysique qui oriente et détermine non seulement la médecine

\footnotetext{
${ }^{51}$ Cf. Aristote, Part. an., X, 655b5-7 ; Eth. Nic., I, 8, $1098 \mathrm{~b} 21$.

52 Cf. Aristote, Eth. Nic., I, 3, 1095 b18.

53 Cf. Heidegger, GA 18 (1924), p. 202 : « Le noein de l'homme n'est pas pur ».
} 
psychiatrique, mais aussi la psychologie, la psychophysiologie, la psychopathologie, voire la psychanalyse.

Arrêtons-nous à ce stade précis où il faudrait maintenant examiner plus en détail comment cette destruction critique, c'est-à-dire cette réappropriation de la «psychologie» aristotélicienne, lue par Heidegger comme une "ontologie du vivant», permet éventuellement de reconsidérer et d'approfondir le problème du Leib tel qu'il s'esquisse, un peu elliptiquement et comme par réminiscence, dans les Séminaires de Zollikon, tout en interrogeant, sous cet angle, les conséquences de cette reconstruction pour l'élaboration d'une Daseinsanalyse qui s'en réclamerait. 